\title{
Analysis of slab bridges using boundary element method
}

\author{
E. M. Galuta \& M. Taghdi \\ Al Fateh University, Tripoli, Libya
}

\begin{abstract}
This paper presents the advantages of using Boundary Element Method (BEM) as a numerical technique, compared with the other numerical methods such as a Finite Element Method (FEM), to analyze three dimensional problems such as bridges. The mathematical formulation of the boundary element equation is also presented. Analyzing the bridge deck by BEM would be more efficient than by FEM, where only the boundaries along the bridge deck have to be discretized into one-dimensional elements.Therefore, the dimensions of the analysis are reduced, and consequently the input data is significantly reduced. For bridges subjected to moving loads, it is necessary to subdivide the bridge deck into a number of finer meshes in FEM, thus it leads to huge number of simultaneous equations and large band width. In addition to that the mesh needs to be changed as the truck loads are moving along the bridge deck, while in BEM the moving loads do not affect the size of the mesh and the mesh does not require any change. A very important application of the BEM is that there is no interpolation of the solution within the domain, and for a given solution on the surface, the results at interior points involve no approximations. Two numerical examples were presented to demonstrate the accuracy and the advantages of using BEM over the other numerical methods in the bridge analysis
\end{abstract}

Keywords: bridges, boundary element, bridge deck, finite element, slab bridges, mesh.

\section{Introduction}

In the analysis of slab bridges, the most commonly domain-type method used is Finite Element Method. The method can solve complex material properties in a finite domain and can be applied to analyze any types of bridges. However, when 
the method is applied to analyze bridges subjected to moving truck loads, the finite element mesh requires more refinement near the point loads, therefore, this will lead to a huge number of equations. In addition to that the mesh needs to be changed as the truck loads are moving along the bridge deck.

The BEM has recently become a popular and powerful numerical technique to analyze various engineering problems. The main advantage of the method is that the boundary equations need to be applied only to the boundary of the solution domain. Therefore, the dimension of a given problem becomes smaller that that generated by finite element method. Also, for bridges under truck loads, the BEM is capable of simulating the local effect of the truck loads, and its mesh does not require any changes as the loads are moving over the bridge deck

In this paper the boundary element equations for plate bending elements are presented. Two numerical examples for bridge analyses are presented to demonstrate the simplicity and accuracy of the boundary element method compared with the other numerical solutions.

\section{Boundary integral equations}

The boundary integral equations of plates can be established by applying unit force and unit moment at the source point of each node and implementing the Betti-Maxwell theorem, two boundary integral equations can be written for every node as follow [1]:

$$
\begin{aligned}
C_{b} w_{p} & +\oint_{s}\left(W(s i) S^{f z}+\theta(s i) M^{f z}\right) d s+\sum_{i=1}^{N_{c}} W_{i} \Delta T_{i}^{f z}=\iint_{A} q w^{f z} d A \\
& +\int_{s}\left(S(s i) W^{f z}+M(s i) \theta^{f z}\right) d s+\sum_{i=1}^{N_{c}} \Delta T_{i} W_{i}^{f z} \\
C_{b} \theta_{p}^{m \xi}+ & \oint_{s}\left(W(s i) S^{m \xi}+\theta(s i) M^{m \xi}\right) d s+\sum_{i=1}^{N_{c}} W_{i} \Delta T_{i}^{m \xi}=\iint_{A} q w^{m \xi} d A \\
& +\int_{s}\left(S(s i) W^{m \xi}+M(s i) \theta^{m \xi}\right) d s+\sum_{i=1}^{N_{c}} \Delta T_{i} W_{i}^{m \xi}
\end{aligned}
$$

where $W, \theta, S$ and $M$ are the vertical deflection, normal slope, equivalent shear force and normal moment at the boundary respectively, see figure $1 . w_{p}$ and $\theta_{p}$ are the deflection and slope inside the plate, $\mathrm{N}_{\mathrm{C}}, \mathrm{A}, \mathrm{q}$, and $\mathrm{s}$ are the number of corners, the loaded area, the vertical distributed load and the plate boundary respectively, $f z$ and $m \xi$ are superscripts referring to force and moment fundamental solutions in the $\mathrm{z}$ and $\xi$ directions respectively, $C_{b}$ is a constant defining the location of the source point $\mathrm{p}[2] . \Delta \mathrm{T}$ is the effective corner force and it is given as:

$$
\Delta T=T^{+}-T^{-}
$$




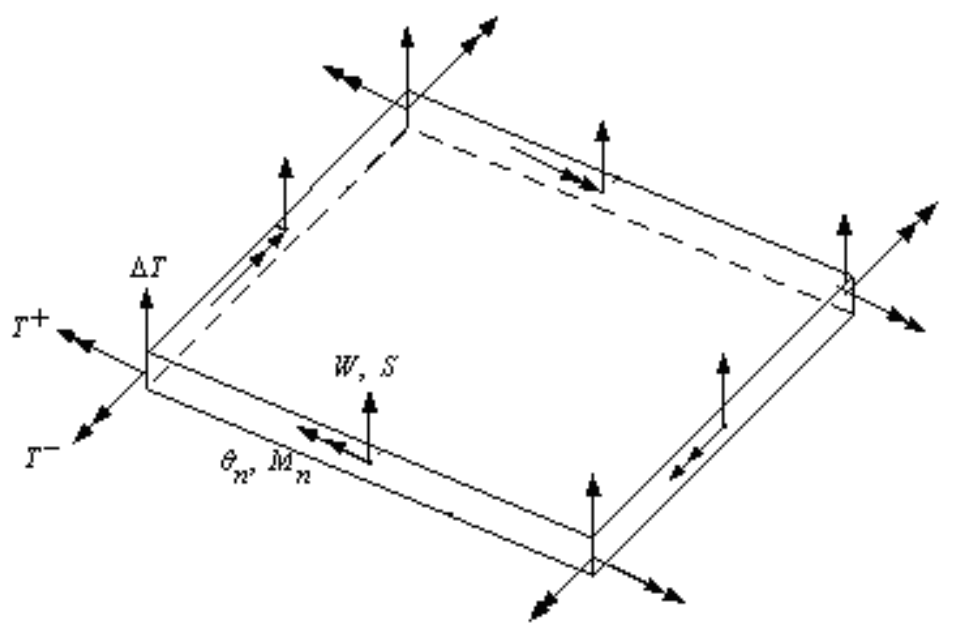

Figure 1: Direction of displacements and forces along the boundaries.

To obtain a numerical solution for eqns (1) and (2), the boundary is divided into a discrete number of elements and the boundary variables (i.e. $W(s) i$ and, $\theta(s i))$ are expressed in terms of their nodal values using Lagrange interpolation functions. After substituting the fundamental solutions into the boundary integral variables and adding the contribution of all elements, a linear algebraic equation can be written in a matrix from as:

$$
[H]\left\{\begin{array}{l}
W \\
\theta
\end{array}\right\}=[G]\left\{\begin{array}{l}
S \\
M
\end{array}\right\}+\{q\}
$$

Where $[\mathrm{H}]$ and $[\mathrm{G}]$ are matrices including the coefficients corresponding to the displacements and the forces respectively, and $\{\mathrm{q}\}$ is a vector including the domain integral. All boundary unknowns in eqn (4) can be solved after imposing the boundary conditions. Then these nodal displacements and forces can be employed in the boundary element equations to calculate the displacements and stresses at any internal points.

\section{Numerical examples}

\subsection{Example 1}

A simply supported concrete slab bridge under two trucks is analyzed to show the advantages of using BEM over FEM in terms of simplicity and reduction of input data. The dimensions, material properties and the idealization of the bridge are shown in figure 2 . In the finite element analysis, the bridge is discretized 


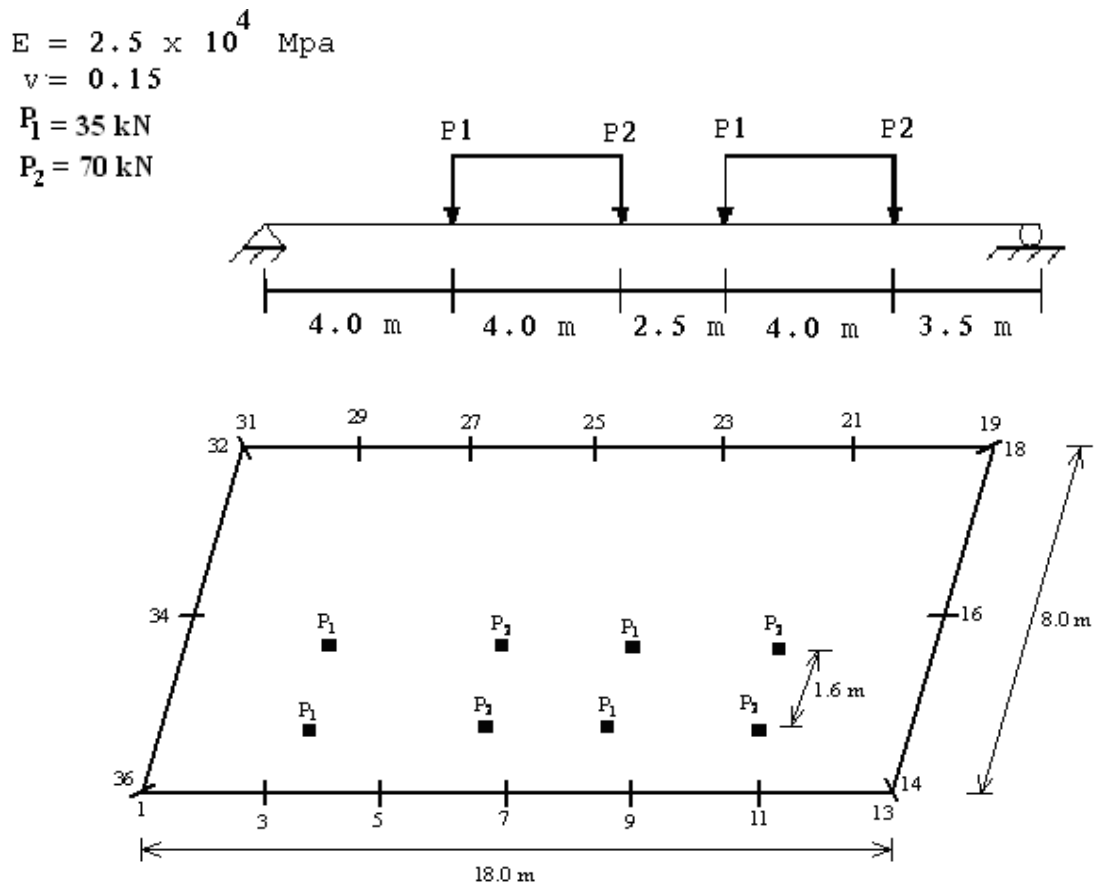

a) Dimension, material properties and BEM mesh

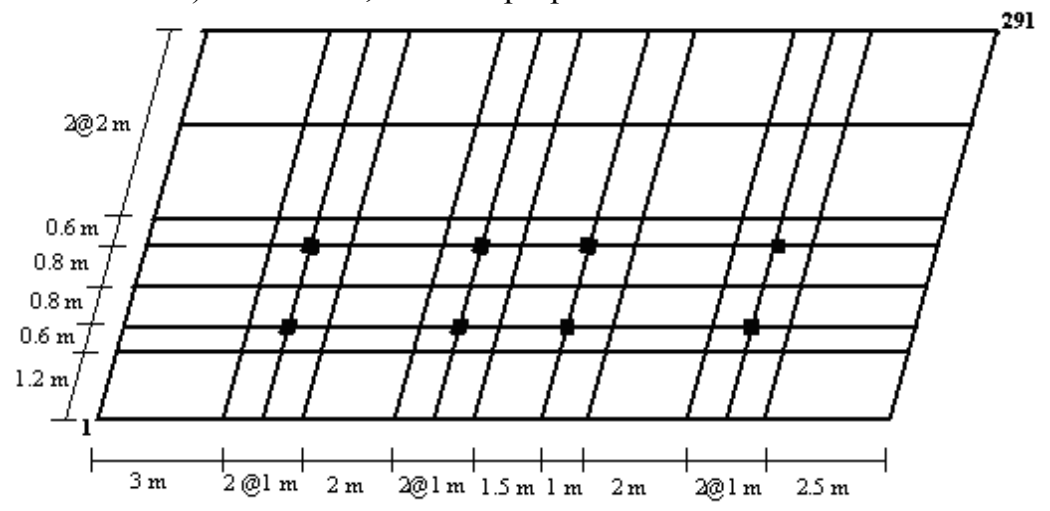

b) FEM mesh

Figure 2: $\quad$ Details of the bridge in example 1.

into 84 rectangular elements with a total of 291 nodes, while the boundary element mesh consisted only of 16 quadratic elements with 36 nodes.

The results of the longitudinal moments (M) and central deflections (W) are given in table 1. As we can see, the BEM is more efficient than FEM when the bridges are subjected to moving loads. The position and number of loads do not 
change the boundary element mesh where only the boundaries need to be discretized, while the finite element mesh needs to be changed as the loads are moving over the bridge. In addition to that, the finite element mesh requires more refinement near the leads, thus it leads to huge number of simultaneous equations and large band width.

Table 1: Vertical deflection and longitudinal moment along the span.

\begin{tabular}{|c|c|c|c|c|}
\hline \multirow{2}{*}{ Span $(\mathrm{m})$} & \multicolumn{2}{|c|}{ BEM } & \multicolumn{2}{c|}{ FEM } \\
\cline { 2 - 5 } & $\mathrm{W}(\mathrm{mm})$ & $\mathrm{M}(\mathrm{kN} . \mathrm{m} / \mathrm{m})$ & $\mathrm{W}(\mathrm{mm})$ & $\mathrm{M}(\mathrm{kN} . \mathrm{m} / \mathrm{m})$ \\
\hline 1.5 & 38.3 & 3.83 & 38.1 & 5.62 \\
3.5 & 85.29 & 10.19 & 84.78 & 13.34 \\
4.5 & 105.3 & 12.22 & 104.7 & 16.38 \\
6.0 & 129.5 & 13.60 & 128.7 & 19.77 \\
7.5 & 145.2 & 19.76 & 144.3 & 23.72 \\
8.5 & 150.2 & 20.78 & 149.2 & 24.72 \\
9.75 & 149.8 & 18.78 & 148.9 & 23.92 \\
11.0 & 142.4 & 17.07 & 141.5 & 22.64 \\
14.0 & 98.8 & 16.69 & 98.0 & 17.50 \\
15.0 & 77.2 & 14.78 & 76.6 & 15.76 \\
16.75 & 33.5 & 4.87 & 33.2 & 5.80 \\
\hline
\end{tabular}

\subsection{Example 2}

A concrete deck of a simply supported box girder bridge is considered in this example. The deck is subjected to uniformly distributed load of $4 \mathrm{kN} / \mathrm{m}$. Material properties, dimension and meshes for the bridge deck are shown in figure 3 . The purpose of this example is to investigate the convergence of the BEM solution by changing the order of the boundary elements. Quadratic, fourth order and sixth order boundary elements were used to model the bridge deck using three different meshes as shown in figures $3 a), 3 b$ ) and $3 c$ ). The convergence of the BEM solution by changing the number of elements with the same order was not investigated in this paper.

The results of the deflection and the longitudinal moment along the centre of bridge deck for the three meshes are compared with the solution of the Finite Strip Method (FSM) in tables 2 and 3.

The results from the two solutions are in good agreement. It is interesting to note that the BEM solution for all meshes almost gave the same results. Therefore, using high order elements in the boundary element idealization is not necessary in order to obtain accurate results. However, high order elements reduce the required input data files. 
$\mathrm{E}=25000 \mathrm{MPa}$

$\mathrm{v}=0.15$

$\mathrm{w}=40 \mathrm{kN} / \mathrm{m}$

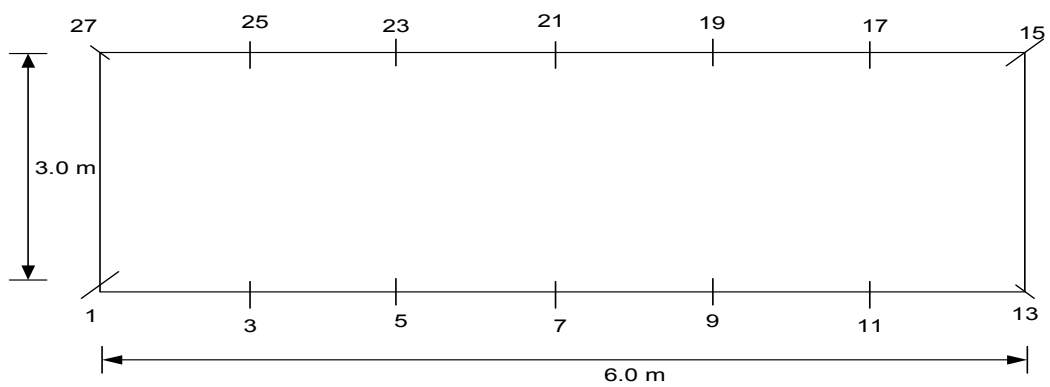

a) Boundary element idealization using quadratic elements.

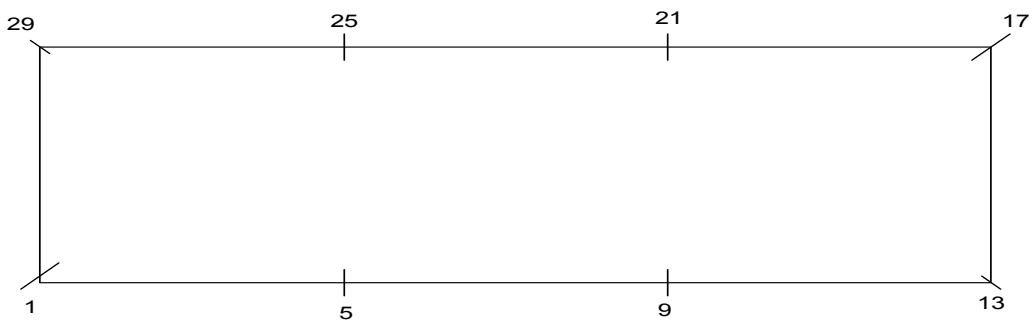

b) Boundary element idealization using fourth-order elements.

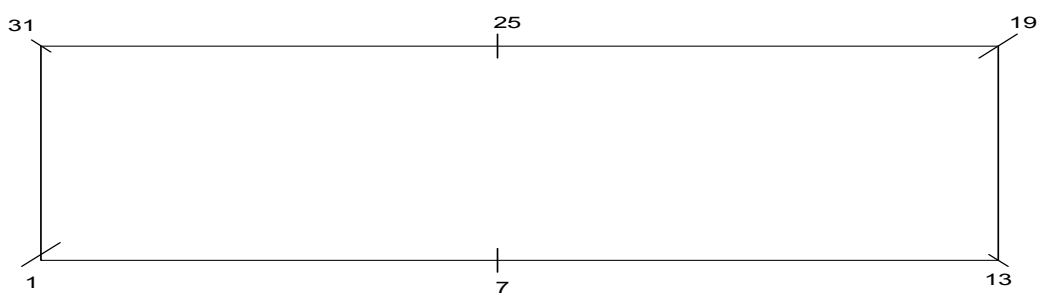

c) Boundary element idealization using sixth-order elements.

Figure 3: $\quad$ Dimension and mesh types for example 2.

Table 2: Vertical deflection along the centre of the span.

\begin{tabular}{|c|c|c|c|c|}
\hline \multirow{2}{*}{$\begin{array}{c}\text { Span }) \\
(\mathrm{m})\end{array}$} & FSM $(\mathrm{mm})$ & \multicolumn{3}{|c|}{ BEM $(\mathrm{mm})$} \\
\cline { 3 - 5 } & & Mesh 1 & Mesh 2 & Mesh 3 \\
\hline 0.5 & 0.189 & 0.202 & 0.193 & 0.191 \\
1.0 & 0.340 & 0.356 & 0.349 & 0.344 \\
1.5 & 0.445 & 0.454 & 0.458 & 0.451 \\
2.0 & 0.510 & 0.509 & 0.528 & 0.519 \\
2.5 & 0.546 & 0.539 & 0.566 & 0.556 \\
\hline
\end{tabular}




\begin{tabular}{|l|l|l|l|l|}
\hline 3.0 & 0.557 & 0.550 & 0.577 & 0.569 \\
& & & & \\
& & & & \\
\hline
\end{tabular}

Table 3: Longitudinal moment along the centre of the span.

\begin{tabular}{|c|c|c|c|c|}
\hline $\begin{array}{c}\text { (Span) } \\
(\mathrm{m})\end{array}$ & FSM (kN.m/m) & \multicolumn{3}{|c|}{ BEM (kN.m/m) } \\
\cline { 3 - 5 } & & Mesh 1 & Mesh 2 & Mesh 3 \\
\hline 0.5 & 7.381 & 7.076 & 7.222 & 7.296 \\
1.0 & 12.780 & 12.505 & 12.819 & 12.783 \\
1.5 & 16.240 & 16.485 & 16.307 & 16.281 \\
2.0 & 18.210 & 18.671 & 18.268 & 18.295 \\
2.5 & 19.180 & 19.455 & 19.312 & 19.287 \\
3.0 & 19.470 & 19.583 & 19.648 & 19.583 \\
\hline
\end{tabular}

\section{Conclusion}

The numerical examples show, in general, the advantages of using the BEM over the other numerical solution to analyze bridges subjected to moving loads. The important design quantities such as longitudinal bending moments and vertical deflection are found to be in good agreement when compared with the other numerical solutions. Using high order elements in the boundary element idealization is not necessary in order to obtain accurate results.

\section{References}

[1] Galuta E.M, "Combined Boundary Element and Finite Analysis of Composite Bridges", Ph.D. Thesis, Ottawa University, (1993).

[2] Brebbia C.A., Telles J.C. and Wrobel L.C., Boundary Elements Techniques: Theory and Application in Engineering, Spinger-Verlag, New York, (1984). 